\title{
Measuring internal quantum efficiency to demonstrate hot exciton dissociation
}

To the Editor - Grancini et al. reported that "hot [charge transfer state] dissociation produces an overall increase in the charge generation yield" in organic solar cells $(\mathrm{OSCs})^{1}$. If true, this is an important result as the process could dramatically improve efficiencies. Hot excitons are a 'hot' topic ${ }^{2,3}$, but was the increase in internal quantum efficiency (IQE) seen by Grancini et al. really due to hot excitons? In this Correspondence we demonstrate that when interference and other optical effects are correctly accounted for, the apparent strong contribution from hot excitons is absent.

Hot excitons are generated by photons with energies greater than the optical gap of organic semiconductors. It has been postulated this extra energy aids separation of bound electron-hole pairs (excitons), leading to an increase in the 'charge generation yield ${ }^{1-3}$. Grancini et al. report signatures of hot electron dissociation in PCPDTBT:PC ${ }_{60} \mathrm{BM}$ OSCs using timeresolved spectroscopy. This central thesis also relies on steady-state measurements on a working solar cell - specifically the observation of higher IQE for higher-energy photons. However, their methodology to calculate IQE does not properly account for the critical optical phenomena of interference and parasitic absorptions in the non-active layers ${ }^{4}$. Organic solar cells are low-finesse cavities in which reflections from the electrodes influence the optical field distribution via interference. These effects impact the measured external quantum efficiency (EQE) and must be accounted for to extract an accurate IQE, which contains information regarding charge generation and collection efficiency versus photon energy ${ }^{5}$. Grancini et al. scaled their EQE by the absorption of the active-layer on glass, which only yields an 'IQE-like' spectrum (Fig. 5b in ref. 1) and not the 'true' IQE.

We have repeated these steady-state measurements in solar cells made from the same junction materials and with a similar architecture (glass/ITO $(80 \mathrm{~nm}) /$ PEDOT:PSS $(20 \mathrm{~nm}) /$ PCPDTBT:PC ${ }_{60} \mathrm{BM}$ $(60 \mathrm{~nm}) / \mathrm{Al}(100 \mathrm{~nm}))$, where ITO is indium tin oxide and PEDOT:PSS is poly(ethylene dioxythiophene:poly(styrenesulphonate). We measured the EQE and then calculated both the IQE-like (ratio of the normalized

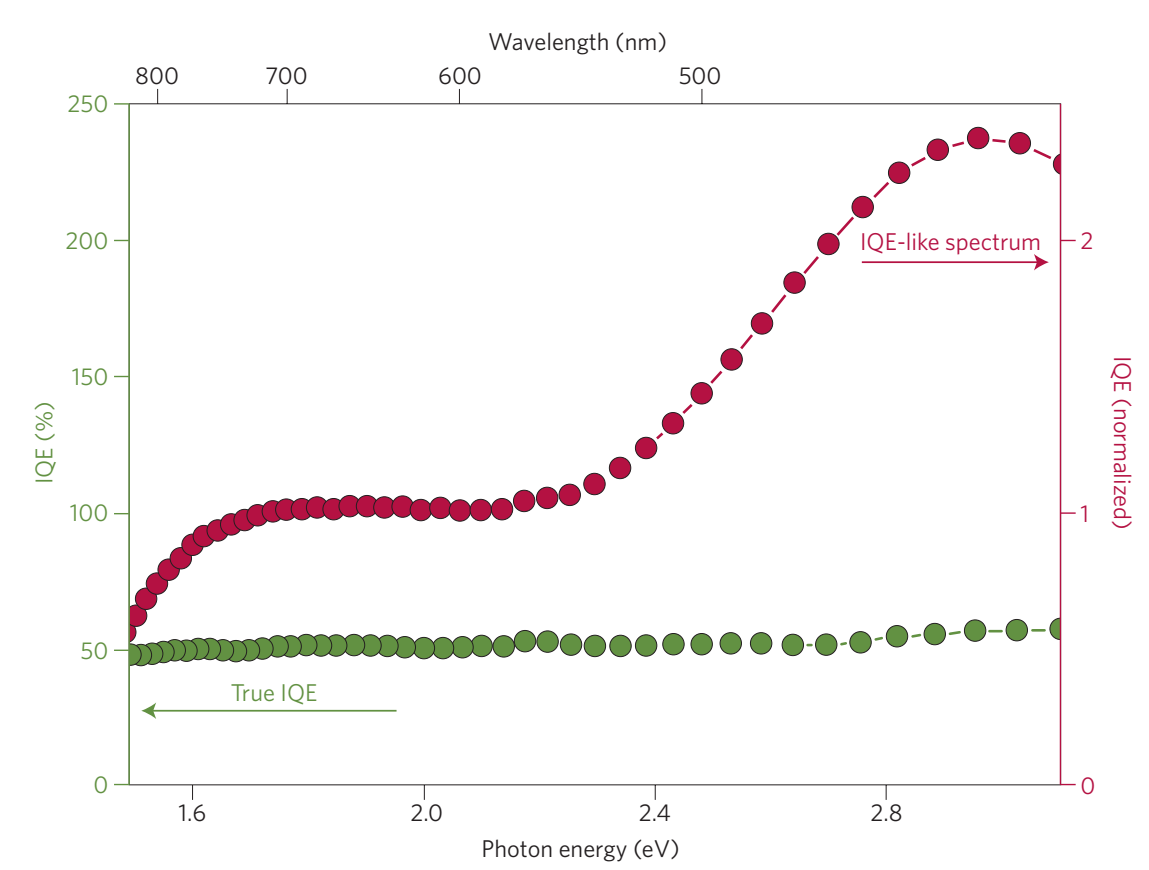

Figure 1 | IQE in OSCs. The true-IQE spectrum taking into account optical interference effects and parasitic absorptions, and the IQE-like spectrum obtained following the procedure reported by Grancini et al. ${ }^{1}$ for the same PCPDTBT:PC ${ }_{60}$ BM device with a junction thickness of $60 \mathrm{~nm}$ (optimized for efficiency). No dependence of the exciton dissociation on photon energy is observed in the true IQE, which is spectrally flat within the measurement certainty ( $5 \%)$. However, and similar to that reported by Grancini etal.', the IQE-like spectrum shows strong photon energy dependence (>200\% increase at $3 \mathrm{eV}$ versus $2 \mathrm{eV}$ ).

EQE and absorption of the active layer as described by Grancini et al.) and trueIQE spectra. To extract the latter, we used ellipsometry to determine the refractive indices of each layer, measured the device reflectance to obtain the absorption of the whole cell, and finally used a transfer matrix approach to extract the parasitic absorptions in the non-active layers. This approach delivers accurate and true-IQE ${ }^{4}$, and should always be deployed in analysing OSCs.

Figure 1 shows a comparison between IQE-like and true-IQE spectra. Similar to Grancini et al., our IQE-like spectrum demonstrates a strong dependence on photon energy. Higher-energy photons seem to create significantly more free carriers. However, the true IQE shows no such dependence - it is essentially flat with no appreciable benefit from higher-energy photons. These clear differences originate from optical interference effects and light absorption in the non-active layers.
Hence, we must conclude that the IQE-like spectrum presented by Grancini et al. does not provide evidence for hot excitons in operational OSCs. Furthermore, this case highlights the importance of treating an OSC as a complex optical cavity as well as a charge-generating device.

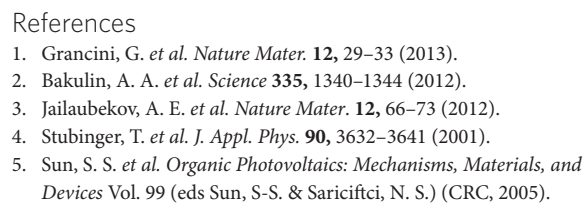

Ardalan Armin, Yuliang Zhang, Paul L. Burn, Paul Meredith and Almantas Pivrikas ${ }^{\star}$ Centre for Organic Photonics \& Electronics (COPE), School of Chemistry and Molecular Biosciences and School of Mathematics and Physics, The University of Queensland, Brisbane 4072, Australia.

*e-mail: almantas.pivrikas@uq.edu.au 